\title{
Cardiovascular Implant Durability Conference (CVID) Rescheduled to April
}

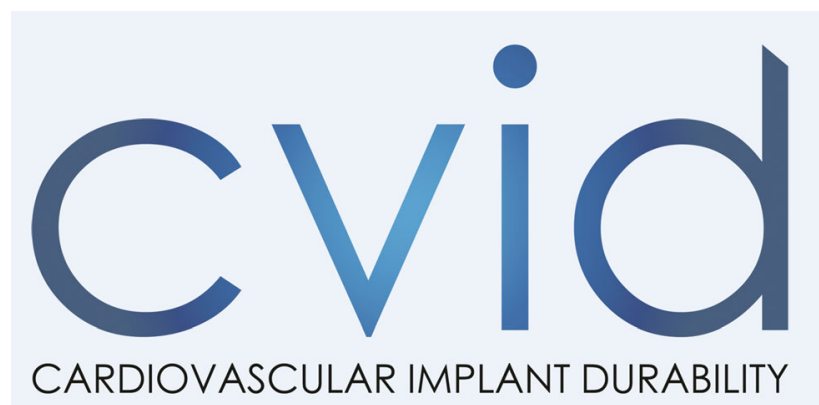

The Cardiovascular Implant Durability Conference, originally intended to take place in October 2021, has been rescheduled to April 4-6, 2022. Organizers stated that "postponing will allow us to provide a safe experience with richer interactions." Shape Memory and Supereleasticity associate editor Tom Duerig and editorial board member Alan Pelton, along with other renowned experts in the field, are part of the organizing committee.

This event is intended to link anatomic and physiological conditions to implant design and to the engineering mechanics of fracture. CVID brings together experts in the fields of medicine, biomechanics, computational analysis, and engineering testing from the medical device industry, academia, physicians, and regulatory agencies.

Shape Memory is also pleased to announce that a special issue based on topics from this conference is scheduled for publication in 2022. Organized by guest editors Dr. Harshad Paranjape of Confluent Medical Technologies and Dr.
Srinidhi Nagaraja of GRAU, Inc., with the assistance of Dr. Duerig and Dr. Pelton, the special issue will feature review articles on very high cycle fatigue of Nitinol, preprocessing effects on Nitinol fatigue, advances in Nitinol modeling enabled by data-driven methodologies, boundary conditions and long-term implantation effects with cardiovascular Nitinol implants, and Nitinol corrosion and biocompatibility. There is also an open call for solicitation of research articles for this issue. Please refer to https:// www.springer.com/journal/40830/updates/19678730 for details.

Further information on the CVID Conference and registration details can be found here: https://cvidconference. org/

Publisher's Note Springer Nature remains neutral with regard to jurisdictional claims in published maps and institutional affiliations. 